УДК 629.027

DOI: 10.34220/2311-8873-2021-4-4-114-119

МОДЕЛИРОВАНИЕ РЕЖИМА ДВИЖЕНИЯ ГРУЗОВОГО

АВТОМОБИЛЯ С КОМБИНИРОВАННЫМ УПРУГИМ

ЭЛЕМЕНТОМ В ПОДВЕСКЕ ЗАДНЕГО МОСТА

Прядкин В.И., Завьялов А.М.

Федеральное государственное бюджетное образовательное учреждение высшего образования «Воронежский государственный

лесотехнический университет им. Г.Ф. Морозова»

E-mail: vip16.vgltu@mail.ru

Аннотация: В статье проведён анализ упругих элементов с нелинейной характеристикой. Для повышения плавности хода грузового автомобиля предложен комбинированный упругий элемент, включающих полуэлептическую рессору и пневматический баллон, для установки в подвеске заднего моста автомобиля. С целью оценки влияния комбинированного упругого элемента с нелинейной характеристикой на плавность хода разработана математическая модель. Математическая модель позволяет проводить численных эксперимент с учетом характера взаимодействия шин с неровностями дорожного полотна и нелинейной характеристики комбинированного упругого элемента.

Ключевые слова: подвеска, нелинейная упругая характеристика, динамическая система, жесткость, масса.

\title{
SIMULATION OF TRUCKING \\ MODE CARS WITH A COMBINED ELASTIC \\ ELEMENT IN THE REAR AXLE SUSPENSION \\ Pryadkin V.I., Zavyalov A.M.
}

Federal State Budgetary Educational Institution of Higher Education «Voronezh State Forestry University. G.F. Morozova»

E-mail: vip16.vgltu@mail.ru

Summary: The article analyzes elastic elements with a nonlinear characteristic. To improve the smoothness of a truck, a combined elastic element is proposed, including a semi-eleptic spring and an air cylinder, for installation in the rear axle suspension of a car. In order to assess the effect of a combined elastic element with a nonlinear characteristic on the smoothness of the ride, a mathematical model has been developed. The mathematical model allows for a numerical experiment taking into 
account the nature of the interaction of tires with unevenness of the roadway and the nonlinear characteristics of the combined elastic element.

Keywords: suspension, nonlinear elastic characteristic, dynamic system, stiffness, mass.

Одним из способов повышения плавности хода автомобилей является использование упругих элементов с нелинейной упругой характеристикой в подвеске. Для реализации нелинейной характеристики используют различные виды упругих элементов: металлические упругие элементы в виде цилиндрических витых пружин с переменным шагом, комбинированные, включающие полуэлептическую рессору и подрессорник; пневматические - одноступенчатые и двух ступенчатые, а также с противодавлением; гидропневматические и резиновые [1, 2].

Мобильное средство МЭС-90СХ было оборудовано комбинированным упругим элементом, включающим полуэллиптическую рессору с линейной характеристикой и пневматический упругий элемент с нелинейной характеристикой. При малых нагрузках рессора обеспечивает большой ход, а при увеличении нагрузки вступает в работу пневматический упругий элемент, что обеспечивает реализацию нелинейной характеристики в подвеске ведущего моста.

Пневматические элементы в подвеске обеспечивают нелинейную силовую характеристику подвески, а при использовании эффекта дросселирования в совокупности с внешними пневматическими элементами обеспечивают дополнительные демпфирующие свойства $[3,4]$. Пневматические элементы в подвеске обеспечивают устранение резонансных колебаний корпуса автомобиля при движении по существенно неровной опорной поверхности. Для обоснования параметров пневматического элемента в подвеске целесообразно использовать математическое моделирование.

В рамках данной работы подвеска автомобиля представляется одномерной двухмассовой моделью (рис. 1), имитирующей задний мост автомобиля. Автомобиль движется по неровной опорной поверхности, которая задается возмущающей функцией $z_{д}(t)$. Несмотря на простоту, модель позволяет передать основные особенности работы подвески с пневматическим элементом, проверить ее эффективность для различных параметров неровностей опорной поверхности, определить оптимальные параметры пневматического элемента [5-8].

Задние колеса автомобиля приведены в модели к точечной массе $m$. Кор- 
пус автомобиля считается условно приведенным к заднему мосту и заменяется точечной массой $M$, к которой приведена часть массы автомобиля в зависимости от геометрической конфигурации автомобиля и расположения центра тяжести. Материальные точки, представляющие колесо и корпус автомобиля, могут двигаться вдоль оси $O Z$ и задаются координатами соответственно $z_{\mathrm{m}}$ и $z_{\mathrm{M}}$. Шина задается упруго-вязким взаимодействием между опорной поверхностью и центром колеса. Упругое и вязкое взаимодействия характеризуется коэффициентами соответственно жесткости $c_{\text {ш }}$, и демпфирования $d_{ш}$. По мере движения коле-

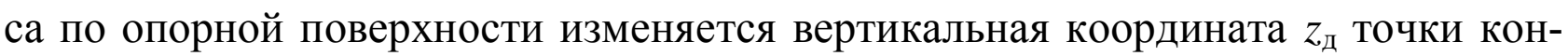
такта с опорной поверхностью. Появление упруго-вязких сил между опорной поверхностью и центром колеса приводит к движению центра масс колеса $m$, что, в свою очередь, приводит к движению корпуса автомобиля $M$.

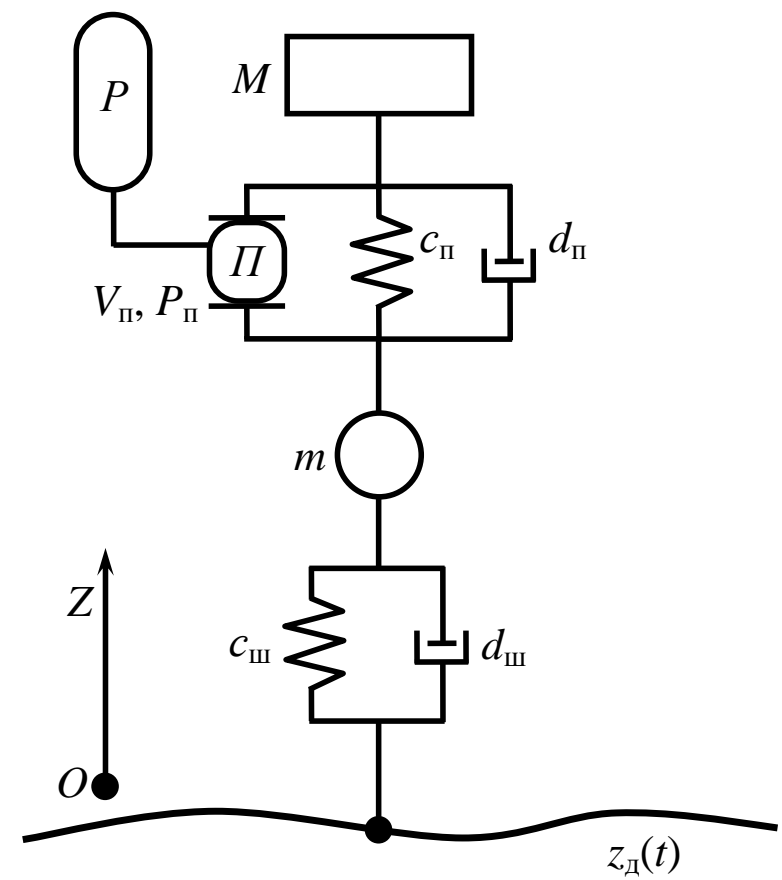

Рисунок 1 - Представление автомобиля в модели в виде двухмассовой системы с пневматическим элементом в подвеске

Между центром масс колеса $m$ и точкой крепления к корпусу $M$ действуют силы, возникающие в подвеске. Подвеска состоит из трех элементов. Рессоры представляют собой упругий элемент, характеризующийся в модели коэффициентом жесткости $c_{\text {п }}$. Амортизатор в модели считается линейным демпфе-

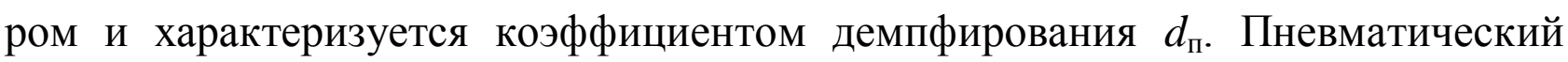
элемент «П» характеризуется объемом $V_{\text {п }}$ и давлением газа $P_{\text {п }}$.

Уравнения динамики автомобиля записаны в соответствии со вторым законом Ньютона: 


$$
\left\{\begin{array}{l}
\frac{d^{2} M}{d t^{2}}=-M g+c_{n}\left(z_{a 0}-\left(z_{M}-z_{m}\right)\right)-d_{n}\left(\frac{d z_{M}}{d t}-\frac{d z_{m}}{d t}\right)+P_{n} S_{n} \\
\frac{d^{2} m}{d t^{2}}=-m g-c_{n}\left(z_{a 0}-\left(z_{M}-z_{m}\right)\right)+d_{n}\left(\frac{d z_{M}}{d t}-\frac{d z_{m}}{d t}\right)+ \\
+c_{u}\left(z_{m 0}-\left(z_{m}-z_{\partial}(t)\right)\right)-d_{u} \frac{d z_{m}}{d t}-P_{n} S_{n} \\
P_{n}\left(V_{p}+S_{n}\left(z_{a 0}-\left(z_{M}-z_{m}\right)\right)\right)^{\frac{7}{5}}=\text { const }
\end{array}\right.
$$

где $M$ и $m$ - массы корпуса и колеса, приведенные к заднему мосту; $t$ - время; $g$ - ускорение свободного падения; $c_{\text {п }}$ - коэффициент жесткости рессоры; $c_{\text {ш }}-$ коэффициент жесткости шины; $z_{\mathrm{a} 0}$ - равновесное расстояние между точками $m$ и $M ; z_{\mathrm{M}}$ и $z_{\mathrm{m}}-$ координаты центра масс колеса и точки крепления подвески к корпусу автомобиля; $d_{\text {п }}$ и $d_{\text {щ }}-$ коэффициенты демпфирования упругого элемента подвески и шины; $z_{\mathrm{m} 0}$ - равновесная координата центра масс колеса; $z_{\text {д }}$ координата точки контакта колеса с опорной поверхностью; $P_{\text {п }}$ - давление газа в пневматическом элементе; $S_{\text {п }}$ эффективная площадь пневматического элемента; $V_{\mathrm{p}}$ - объем ресивера.

Последнее уравнение системы представляет собой уравнение состояния газа в пневматической системе в рамках адиабатического приближения и позволяет рассчитать силу в подвеске со стороны пневматического элемента в зависимости от его деформации. Также, в модели принято допущение, что давление газа в пневматическом элементе равно давлению газа в ресивере, то есть газопровод между пневматическим элементом и ресивером не оказывает дросселирующего действия.

Данная система дифференциальных уравнений второго порядка в общем случае не имеет аналитического решения, в частности, из-за необходимости исследовать разнообразные возмущающие функции $z_{д}(t)$. Поэтому для решения данной системы дифференциальных уравнений используется универсальный численный метод Рунге-Кутта второго порядка. Численное решение дифференциальных уравнений заключается в дискретизации времени $t$ на равные шаги, нумеруемые переменной $\tau$, с длительностью шага $\Delta t$. На каждом шаге интегрирования рассчитываются силы $F_{\mathrm{m} \tau}$ и $F_{\mathrm{M \tau}}$, действующие на тела механической системы, то есть правые части дифференциальных уравнений (1). После этого по известным координатам и скоростям движения тел на текущем шаге интегрирования рассчитываются координаты и скорости тел на следующем шаге интегрирования: 


$$
\left\{\begin{array}{l}
z_{m \tau+1}=z_{m \tau}+v_{m \tau} \cdot \Delta t+\frac{F_{m \tau}}{m} \cdot \frac{(\Delta t)^{2}}{2} ; \\
z_{M \tau+1}=z_{M \tau}+v_{M \tau} \cdot \Delta t+\frac{F_{M \tau}}{M} \cdot \frac{(\Delta t)^{2}}{2} \\
v_{m \tau+1}=v_{m \tau}+\frac{F_{m \tau}}{m} \cdot \Delta t \\
v_{M \tau+1}=v_{M \tau}+\frac{F_{M \tau}}{M} \cdot \Delta t
\end{array}\right.
$$

где $z_{\mathrm{m} \tau}, z_{\mathrm{M} \tau}, v_{\mathrm{m} \tau}, v_{\mathrm{M} \tau}$ - координаты и скорости движения вверх тел $m$ и $M$ на предыдущем шаге интегрирования по времени $\tau ; z_{\mathrm{m} \tau}, z_{\mathrm{M} \tau}, v_{\mathrm{m \tau}}, v_{\mathrm{M \tau}}-$ то же, на последующем шаге интегрирования по времени $\tau+1$. Параллельно, на каждом шаге интегрирования по времени просчитываются параметры состояния газовой системы $P_{\text {пг }}$ и $V_{\text {пт }}$. По мере пересчета предыдущих координат и скоростей в последующие, получаются таблично заданные функции зависимости от времени координат $z_{\mathrm{m}}(t), z_{\mathrm{M}}(t)$, которые анализируются далее для оценки плавности хода автомобиля.

Случайная неровная поверхность представляется в виде суперпозиции определенного количества гауссовых пиков:

$$
z_{\partial}(t)=\sum_{i=1}^{N_{\mu}} H_{i} \exp \left(-\frac{\left(v \cdot t-x_{i}\right)^{2}}{2 \sigma_{i}^{2}}\right)
$$

где $N_{\text {н }}$ - количество неровностей гауссовой формы на контрольном участке заданной длины $L_{\mathrm{\kappa}} ; H_{\mathrm{i}}-$ высота $i$-й неровности; $x_{\mathrm{i}}$ - координата центра $i$-й неровности; $\sigma_{\mathrm{i}}$ - характерная ширина $i$-й неровности (имеет смысл среднеквадратичного отклонения); $v$ - скорость горизонтального движения автомобиля.

Для оценки плавности хода автомобиля рассчитываются спектры колебаний автомобиля в вертикальном направлении $A_{z}(f)$ (амплитудно-частотные характеристики - АЧХ). Для расчета АЧХ производится Фурье-преобразование функци $z_{\mathrm{M}}(t)$ следующим образом:

$$
A_{z}(f)=k_{\mu} \sqrt{\left(\int_{0}^{t_{K \ni}} z_{M}(t) \sin (2 \pi f t) d t\right)^{2}+\left(\int_{0}^{t_{K \ni}} z_{M}(t) \cos (2 \pi f t) d t\right)^{2}},
$$


где $k_{\text {н }}$ - нормировочный коэффициент, определяющий общий уровень колебаний; $f$ - частота колебаний. Так как функция $z_{\mathrm{M}}(t)$ задана таблично, интегралы в формуле для спектра рассчитываются численным методом - методом прямоугольников.

Таким образом, разработана достаточно адекватная модель движения грузового автомобиля, оснащенного подвеской с пневматическим элементом, позволяющая изучить влияние параметров пневматического элемента и рельефа опорной поверхности на плавность хода автомобиля.

\section{СПИСОК ЛИТЕРАТУРЫ}

1 Раймпель Й. Шасси автомобиля : Конструкции подвесок / Пер. с нем. В. П. Агапова. - М. : Машиностроение, 1989. - 328 с.

2 Раймпель Й. Шасси автомобиля : Элементы подвески / Пер. с нем. А. Л. Карпухина ; под ред. Г. Г. Гридасова. - М. : Машиностроение, 1987. - 288 с.

3 Прядкин, В. И. Транспортно-технологические средства на шинах сверхнизкого давления / В. И. Прядкин, В. Я. Шапиро, З. А. Годжаев, С. В. Гончаренко ; М-во образования и науки РФ, ФГБОУ ВО «ВГЛТУ». - Воронеж, 2019. $-492 \mathrm{c}$.

4 Прядкин, В. И. Мобильные средства химизации грузоподъемностью 1 ... 2 т на шинах сверхнизкого давления / В. И. Прядкин ; М-во образования и науки РФ, ФГБОУ ВО «ВГЛТУ». - Воронеж, 2017. - 183 с.

5 Зайцев, С. Д. Тягово-сцепные качества высокоэластичных шин сверхнизкого давления / С. Д. Зайцев, С. В. Гончаренко, Л. С. Стреблеченко, В. И. Прядкин, А. Б. Костин // Тракторы и сельскохозяйственные машины. - 2008. - № 9. - C. 29-31.

6 Годжаев, 3. А. Выбор параметров шин сверхнизкого давления для мобильных средств химизации / 3. А. Годжаев, А. Ю. Измайлов, В. И. Прядкин // Тракторы и сельхозмашины. - 2014. - № 4. - С. 14-17.

7 Прядкин, В. И. Шины сверхнизкого давления для сельскохозяйственных мобильных средств / В. И. Прядкин, С. В. Гончаренко ; М-во образования и науки РФ, ФГБОУ ВО «ВГЛТУ». - Воронеж, 2016. - 240 с.

8 Бычков, Н.И. Энергосредство для механизации работ на поймах / Н. И. Бычков, В. И. Прядкин, А. Г. Мельник// Тракторы и сельхозмашины. - 2004. № $10 .-$ C. 6-8. 